PERSPECTIVE

\title{
Bioengineered angiotensin-converting-enzyme-2: a potential therapeutic option against SARS-CoV-2 infection
}

\author{
Biplab K. Maiti iD ${ }^{1 凶}$ \\ (C) The Author(s), under exclusive licence to Springer Nature Limited 2021
}

The recombinant soluble human angiotensin-converting enzyme 2 (rshACE2) is a promising therapy against SARS-CoV-2 infection, but it has some drawbacks that reduce the success of its clinical applications. The bioengineered ACE2 (Tag-sACE2 and probioticACE2) as a way may overcome its therapeutic limitations against ongoing current pandemic.

Journal of Human Hypertension (2022) 36:488-492; https://doi.org/10.1038/s41371-021-00636-y

\section{INTRODUCTION}

A recent outbreak of novel severe acute respiratory syndrome coronavirus 2 (SARS-CoV-2) causes a fatal coronavirus disease 2019, namely COVID-19, that ruins the enormous economy and human health around the globe. Several clinical reports have suggested that COVID-19 patients with weakened immune system and comorbidity are at high risk [1]. On an urgent basis, several pharmaceuticals are being tested clinically, but no one has fruitful outcomes [2]. Therefore, a suitable drug/therapy is required to halt this uninterrupted COVID-19 pandemic.

The primary cause of SARS-CoV-2 infection is hACE2 (human angiotensin-converting enzyme-2) mediated virus binding and enabling entry into the host cell, resulting in dysfunction of multiorgan [3]. However, hACE2 does not play only as a SARS-CoV-2 receptor, but it is well known as a key regulator of lung function [4]. Therefore, hACE2 can be considered as an important target for drug development against COVID-19 pandemic. Multiple attempts are ongoing to halt SARS-CoV-2 infection by the disruption of ACE2-viral interlock. Among them, injection of recombinant soluble form of ACE2 (rSACE2) is a promising approach that can significantly block the virus entry into the host cell and restore the native ACE2 activity also. Several preclinical reports have suggested that rshACE2 therapy may be beneficial for COVID-19 treatment [5], but it has some drawbacks such as short half-life, moderate binding affinity, degradation at acidic $\mathrm{pH}$, and low bioavailability in the target cell, which can retard the success of its clinical applications. Therefore, this concise review aims to explore the bioengineered ACE2 including Tag-SACE2 and probiotic-ACE2, as a way that may overcome therapeutic limitations of ACE2 against SARS-CoV-2 infection.

\section{DUAL ROLE OF ACE2}

The ACE2, a membrane-bound Zn-containing metalloenzyme, is a mono-carboxypeptidase that is counter-balance with its homolog angiotensin-converting enzyme (ACE; known as dipeptidylcarboxypeptidase). Both ACE and ACE2 are key components of renin-angiotensin system (RAS) that regulates a series of physiological and pathophysiological functions, including cardiovascular, and pulmonary systems $[4,6,7]$. In spite of the presence of the same Zn-catalytic site, both ACE2 and ACE show different cleavage patterns, due to the presence of only $40 \%$ amino acid sequence similarity in their catalytic domain $[4,6,7]$. ACE converts decapeptide (Ang-I) to octapeptide (Ang-II), contributing high blood pressure, while ACE2 converts Ang-ll to blood vessel protected heptapeptides (Ang 1-7), resulting in negative effect on RAS, suggesting a tight link between ACE2 (Ang 1-7) and ACE (Ang-II) (Fig. 1).

The hACE2 has been also implicated as a receptor of SARS-CoV2 , enabling cellular entry of virus, thus causing cell toxicity (Fig. 1) [8]. This toxic effect may alter the ACE2 expression, resulting in disruption of the subtle balance between ACE and ACE2, leading to multiple organ injuries. Restoration of the balance between the ACE/Ang-II and ACE2/Ang-(1-7) may protect organs from injuries. Indeed, Kuba et al. reported that SARS-CoV-infected mice model showed the downregulation of the ACE2 expression, developing severe lung injury [9]. This result suggests that deficiency of ACE2 expression leads to respiratory dysfunction. Another experimental result from Zhou et al. had suggested that the overexpression of ACE2 allowed more SARS-CoV-2 infection, resulting in more virus load into the host cell [10]. Therefore, ACE2 not only neutralizes the virus but also rescues the lung from injury. ACE2 is expressed in multiple organs including lungs, intestine, kidneys and blood vessels. The kidneys have highest ACE2 levels together with the intestine whereas lungs have low levels. The clinical manifestations of COVID-19 include more common respiratory symptoms and also gastrointestinal symptoms [11].

The SARS-CoV-2 binding motif of hACE2 is away from its catalytic $\mathrm{Zn}$-site that is the outer surface of the N-terminal domain (Fig. 1). In spite of that, the protective role of ACE2 is abolished upon SARS-CoV-2 binding on ACE2, contributing to severe lung injury. The catalytic cleft of ACE2 harbors two subdomainsmembrane-distal subdomain-I, and membrane-proximal-subdomain-Il, connecting by a hinge that controls the movement of two

\footnotetext{
${ }^{1}$ Department of Chemistry, National Institute of Technology Sikkim, Ravangla Campus, Barfung Block, Ravangla Sub Division, South Sikkim 737139, India. email: biplab@nitsikkim.ac.in
}

Received: 28 July 2021 Revised: 3 November 2021 Accepted: 4 November 2021

Published online: 12 November 2021 


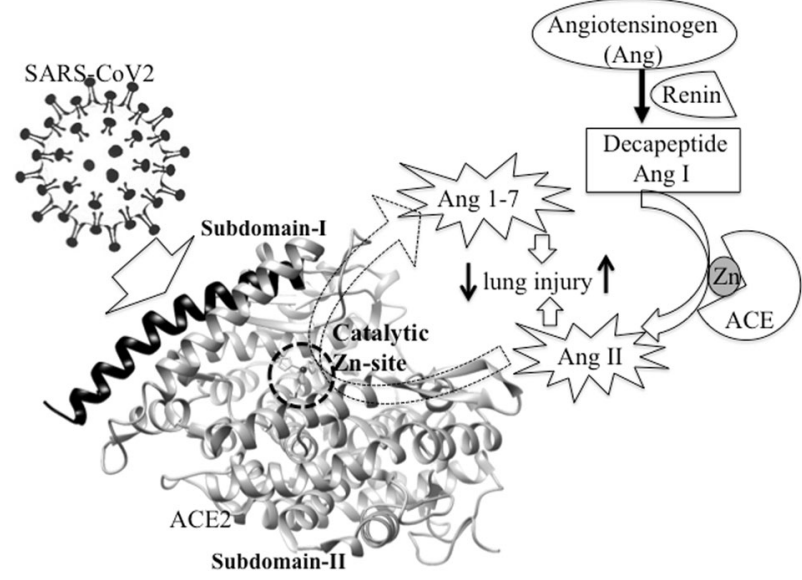

Fig. 1 Dual role of ACE2. Cartoon represents the dual role of ACE2: virus receptor domain (dark black color ribbon) and lung protector catalytic Zn-site (black dotted circle). The ACE2 structure is adopted from PDB-1R42.

domains (Fig. 1) [8]. In the absence of substrate/inhibitor binding, the two subdomains of ACE2 are separated from each other, and thereby the $\mathrm{Zn}$-active site is exposed to solvent, generating an "open-conformation". Therefore, the free state of ACE2 is likely referred to as open-conformation. Upon substrate or inhibitor binding, the subdomain-I moves toward subdomain-II (which remains almost unchanged position), generating "close-conformation". Indeed, a specific inhibitor, MLN-4760 bound ACE2 shows the closed conformation over open-conformation of ACE2 [8]. Upon virus binding on ACE2, the membrane-distal subdomain-I of ACE2 is also slightly moved to membrane-proximal subdomain-II, inducing closed conformation of ACE2 [3]. This subtle conformational change in ACE2 structure may reflect on its protease activity. Recently, Lu et al. have reported that spike bound ACE2 shows a wide range of substrates affinity and speeds up the protease activity with respect to native state in vitro study [12]. This result proposes that subtle conformational change in ACE2 bound SARS-CoV-2 spike protein alters the catalytic activity of ACE2. Therefore, spike bound ACE2 loses the substrate specificity, resulting in more production of Ang-II and Ang 1-7 instead of Ang 1-7 alone [12]. Therefore, homeostatic regulation of the cardiovascular system by virus attached ACE2 is out of controlled, resulting in enhancement of complications.

Functionally, cellular ACE2 exhibits two forms: membranebound ACE2 (mACE2) possessing trans-membrane domain coupled with an exposed extracellular catalytic domain, and soluble ACE2 (sACE2) possessing only catalytic domain. The sACE2 can circulate freely in plasma unlike mACE2, but it shares the same viral binding and enzymatic activity with mACE2 [13]. The cellular ACE2 may be shed by two important cell membrane proteases, ADAM17 (a disintegrin and metalloproteinase 17) and TMPRSS2 (trans-membrane serine protease 2 ), but their cleavage patterns are different (Fig. 2) $[13,14]$. The extracellular region of mACE2 can be trimmed by ADAM17, releasing the SACE2 into the extracellular environment, whereas TMPRSS2 cleaves the ACE2-virus spike complex to facilitate the SARS-CoV-2 entry into the cell for replication. So, mACE2 plays a key role for cellular entry of virus, whereas SACE2 probably has no role for virus entry into the cell. Both cleavage processes reduce the surface density of mACE2, thereby creating significant peripheral imbalance between Ang 1-7 and Ang-II levels that foster organ injuries. Indeed, ACE (AngII) level in plasma of COVID-19-infected patients is markedly elevated compared to healthy persons [15]. This is also found in many other pathological lesions such as diabetes mellitus, hypertension, cardiovascular disease, and ageing [8]. Recently, Kornilov et al. have reported that the formation of SACE2 may be

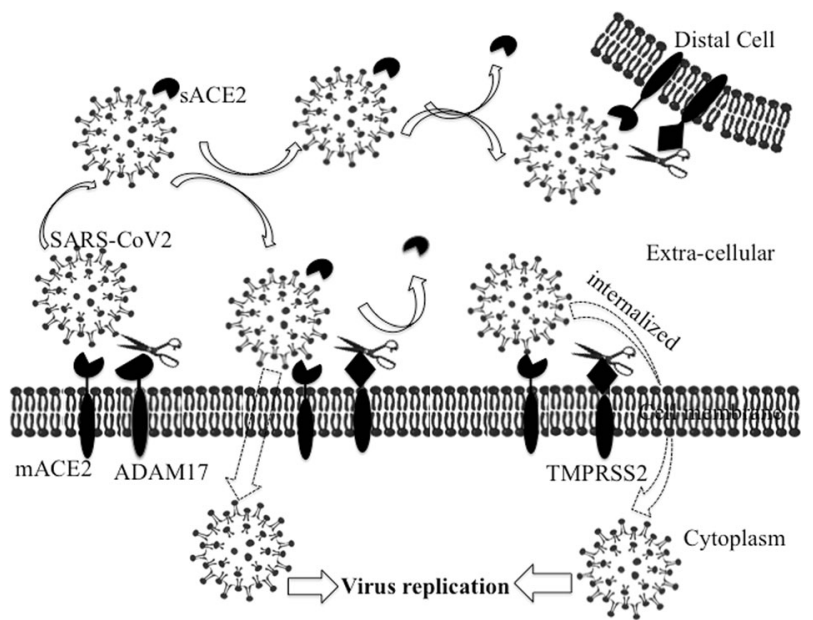

Fig. 2 ACE2 shedding and virus entry \& spreading in cell. Cartoon represents the proposed ACE2 shedding and SARS-CoV-2 entry as well as randomly circulating SACE2-ASRS-CoV-2 complex in extracellular space.

influenced by COVID-19 infection and its concentration may be correlated with degree of inflammation [16]. As concentration of mACE2 at the cell surface is reduced by ADAM17, the degree of virus entry into the cell is also reduced. So, it may be suggested that the initial stage (relatively higher density of mACE2 at cell surface) may be the maximum limit of viral entry into the cell. The sACE2 may bind virus to generate SACE2-coated virus particles, which may freely travel to distant organs, resulting in slow virus spreading and entering into the cell (Fig. 2) [17]. However, sACE2 is a competitor virus receptor with mACE2, and it can be considered as a potential therapy against COVID-19 illness.

\section{ACE2-BASED THERAPY}

\section{Recombinant-sACE2-based therapy}

Several reports have suggested that high levels of rSACE2 may be beneficial for COVID-19 treatment. The proposed mechanism is that the injected rSACE2 may pull the SARS-CoV-2 virus, releasing the $\mathrm{mACE} 2$ as free state, thus reducing the virus load into the cell and maintaining the balance between ACE2 (Ang 1-7) and ACE (Ang-II) (Fig. 3A). In this regard, the clinical grade rsACE2 or hrACE2 is being considered as a promising drug for the treatment of SARS-CoV-2 infection [5]. This sound theoretical idea is appealing for COVID-19 treatment, but needs experimental support. Actually, rSACE2 had been developed previously for the treatment of acute lung injury, respiratory distress syndrome, and hypertension $[18,19]$. After approving by Food and Drug Administration in 2010 , the rsACE2 is being conducted on patients with acute lung injury, respiratory distress syndrome in several clinical phase trials and now also give permission to treat COVID-19 illness due to its rapid transmission and no available registered drug or therapy currently $[18,19]$. Recently, published vitro models and cellular experiments have suggested that therapeutic hrsACE2 or rSACE2 reduces the SARS-CoV-2 entry into the cell and corrects the altered balance of Ang 1-7 and Ang II (Fig. 3A). Indeed, Monteil et al. have reported an interesting result, wherein rshACE2 significantly blocks the SARS-CoV-2 infection in human organoids and VeroE6 cells but required high concentration $(10-200 \mu \mathrm{g} / \mathrm{mL})$ of rshACE2 compared to physiological range in plasma $(\sim \mathrm{ng} / \mathrm{mL})$, providing an important clue regarding drug dose [5]. Of note, a recent and first study on single patient with COVID-19 by Zoufaly et al. has reported that the rshACE2 reduces viral load significantly and has no serious adverse effects or toxicity [20]. This clinical observation encourages it to apply in more clinical trials. 


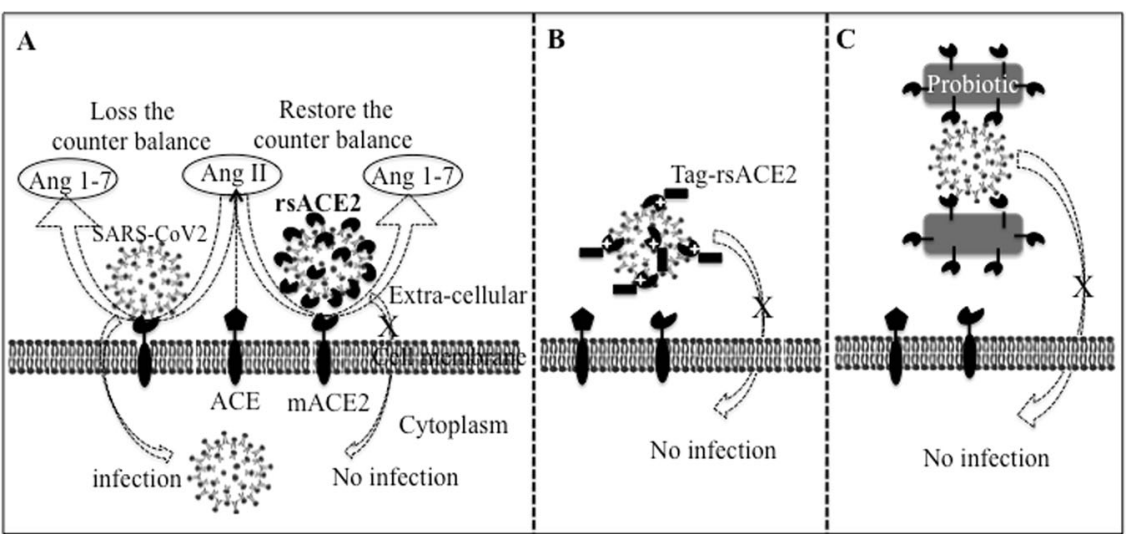

Fig. 3 Bioengineered ACE2: Panel A - rsACE2, Panel B - Tag-rsACE2 and Panel C - Probiotic-ACE2. Probable designed ACE2 for disruption of mACE2-viral interlock: rhACE2 (A), tag-rsACE2 (B), and expression of mACE2 in probiotic (C).

Increased level of rSACE2 at surface of tissue/cell is beneficial for the treatment of COVID-19, but it is opposite to comorbid patients or elderly people who are struggling to get such type of benefit, in spite of the presence of high level of SACE2 in plasma $[15,16]$. This may be the reason for the short half-life of sACE2 [21]. Therefore, rSACE2 may be required to refill the loss of SACE2. Although rSACE2 is currently being studied in clinical trials, it has some limitations that reduce the therapeutic efficacy. The main obstacles of rsACE2 are short half-life, no transport mechanism from circulating to target tissue, moderate binding affinity, enzymatically activation, degradation at low $\mathrm{pH}$, and short-term storage at room temperature, which can be overcome by the protein engineering pathways.

\section{Fusion/bioengineered sACE2-based therapy}

Fusion protein (SACE2) engineering approach offers a promising route to overcome SACE2 limitations. The improvement of the plasma half-life of rSACE2 is desirable for therapeutic use in the clinical setting. To achieve this, rSACE2 is conjugated with a specific tag, human immunoglobulin (lg) crystallizable fragment (Fc) domain. Indeed, Iwanaga et. al., have constructed a cocktail protein, hACE2-IgG1 (Fig. 3B), wherein hACE2-variant coupled with human immunoglobulin $\gamma 1$ Fc region ( $\lg \mathrm{G} 1)$, that shows high plasma stability (half-life $\sim 145$ hs) with excellent bioavailability in the epithelial cell of the lung and reduces the viral load in a COVID-19 infected murine model. However, this is only demonstrated by immunostaining on day 3 post infection [22]. Furthermore, to improve the binding capacity, sACE2-lgG1 has been extended to design multivalent forms (di-, tri-, and tetravalent), by using a self-assembled linker. The engineered multivalent ACE2 derivatives enhance the efficiency of hindering viral entry, thus significant halting SARS-CoV-2 infection in the cell culture [23]. It is noted that Fc-dependent effector functions of IgG can potentially lead to antibody-dependent cellular cytotoxicity, and therefore could potentially support viral infection instead of inhibiting it by binding the Fc-tag to cells like macrophages that express high levels of the Fc receptor [24]. To avoid this effect, albumin-binding domain (ABD) is conjugated with a sACE2 variant to generate a monomeric SACE2-ABD protein, which extends the duration of action up to 3 days in vivo and can neutralize the SARS-CoV-2 infectivity in human kidney organoids [25]. The virus binding capacity of SACE2 fused ABD protein is also improved by dimerization. To achieve this, Hassler et al, have designed a SACE2DDC-ABD derivative, wherein SACE2 variant is coupled with ABD and bridged with a linker, 4-cysteine dodecapeptide (DDC) for dimerization [26]. The dimerized SACE2-DDC-ABD derivative displays higher duration of action and binding affinity compared to monomer. This study using an ABD-fused SACE2 protein is shown to be prevented lethal disease when given preventatively to k18-hACE2 mice, an otherwise lethal model of SARS-CoV-2 infection [26].

As spike-bound SACE2 loses the peripheral balance of Ang-II and Ang 1-7 levels, so prior to injection of rhACE2, the Zn-catalytic site should be catalytically inactivated. The rhACE2 can be inactivated by blocking with suitable inhibitors (MLN-4760) [11] or engineering amino acid residues at $\mathrm{Zn}$-site without any significant alteration of virus receptor role [22]. Therefore, high clinical dose of rSACE2 does not affect on protease activity. Indeed, Iwanaga et. al. have designed an ACE2-IgG1 derivative with mutations in the catalytic Zn-site of ACE2 that inactivates the protease activity but significantly increases the virus binding affinity [22].

\section{Probiotic-expressing ACE2-based therapy}

The embedded ACE2 in probiotics may be another promising therapeutic approach that may significantly halt the current SARSCoV-2 outbreak (Fig. 3C). Probiotics are live microbes that are generally involved in various activities in human, such as boosting the immune response against invading pathogens and preventing inflammation [27, 28]. However, the gastrointestinal tract contains a large number of microbiota compared to lung, but a bidirectional communication is present between them, resulting in migration of microbiota from gut to lung, thereby maintaining the gut and lung microbiota composition [28]. The imbalance of gut microbiota (dysbiosis) causes gastrointestinal inflammation, which results in respiratory pathological lesions through this bidirectional communication [29]. Several studies have reported that intestinal infection by SARS-CoV-2 may cause microbiota dysbiosis contributing inflammatory response in patients with severe COVID-19 [30, 31]. This microbiota dysbiosis can be recovered by consumption of particular probiotic strains (commonly used Lactobacillus and Bifidobacteria spp) that may improve immune activity, and thereby reduce the SARS-CoV-2 infection [32]. Recently, bioengineered probiotics with specific strains have been proposed for anti-coronavirus drugs or vaccines delivery vehicle with high therapeutic efficacy, rather than direct treatment on SARS-CoV-2 infection [32]. Bioengineering probiotics have been successfully used for the treatment of ulcerative colitis [33], and irritable bowel syndrome [34]. Furthermore, ACE2 expresses and suppresses inflammation in the human gut where gut microbiota play a key role for the regulation of colonic ACE2 expression, contributing to the gut microbiota-mediated protective role of ACE2 against invading pathogens [35]. Several data have reported that the protective role of microbiota coupled ACE2 
may be abolished during SARS-CoV-2 infection, resulting in intestinal microbiota dysbiosis and ACE2 down-regulation [35]. In addition, therapeutic ACE2 has limitations in oral administration due to the degradation of ACE2 in the acidic environment of the gastrointestinal tract. To overcome the above problems, probioticexpressing ACE2 would be a promising technology against invading pathogens. Recently, Verma et. al. have designed a live vector by embedding ACE2 in probiotic species (Lactobacillus paracasei) to serve as oral delivery of ACE2 with microbial richness in tissues for the treatment of diabetic retinopathy [36]. In addition, Buford et al. have designed a oral delivery $L$. paracase $i$ expressing Ang(1-7) that markedly enhances circulating concentrations of Ang(1-7) in rats [37]. These results give an evidence for feasibility of probiotic-ACE2 regarding delivery, bioavailability, stability, immune response and oral administration. Therefore, it would be a promising drug that may increase the ACE2 level at cell surface as well as control the microbiota dysbiosis in lung and gut via bidirectional pathway, thereby blocking viral entry in lung and intestine cells. Although there is a lack of clinical evidence yet, probiotic-ACE2 could be considered as a next generation drug for the treatment of ongoing COVID-19 pandemic.

\section{CONCLUSION}

In conclusion, SARS-CoV-2 not only plugs on surface receptor protein but also hijacks enzymatic activity of ACE2. The administration of rSACE2 or Tag-rSACE2 or probiotic-ACE2 would be a promising chelation therapy that interferes the virus-ACE2 cross talk and restores the RAS homeostasis, but they may also be recognized by other biomolecules that could interact with ACE2 resulting in negative effect. However, virus is evolving with time, but human ACE2 is not evolving to recognize SARS-CoV-2. Therefore, manipulation of ACE2 or coupled with a suitable tag or probiotic would be a promising approach to develop a new full potent ACE2 drug, which can efficiently solve the current global health crisis.

\section{REFERENCES}

1. Li Q, Guan X, Wu P, Wang X, Zhou L, Tong Y, et al. Early transmission dynamics in Wuhan, China, of novel coronavirus-infected pneumonia. N Engl J Med. 2020;382:1199-207.

2. Gordon $\mathrm{DE}$, Jang $\mathrm{GH}$, Bouhaddou $\mathrm{M}$, Xu J, Obernier $\mathrm{K}$, White $\mathrm{KM}$, et al. A SARSCoV-2 protein interaction map reveals targets for drug repurposing. Nature. 2020;538:459-68

3. Lan J, Ge J, Yu J, Shan S, Zhou H, Fan S, et al. Structure of the SARS-CoV-2 spike receptor-binding domain bound to the ACE2 receptor. Nature. 2020;581:215-20.

4. Guy JL, Lambert DW, Warner FJ, Hooper NM, Turner AJ. Membrane-associated zinc peptidase families: Comparing ACE and ACE2. Biochim Biophys Acta. 2005;1751:2-8.

5. Monteil V, Kwon H, Prado P, Hagelkruys A, Wimmer RA, Stahl M, et al. Inhibition of SARS-CoV-2 infections in engineered human tissues using clinical-grade soluble human ACE2. Cell. 2020;181:905-13.

6. Kuba K, Imai Y, Ohto-Nakanishi T, Penninger JM. Trilogy of ACE2: A peptidase in the renin-angiotensin system, a SARS receptor, and a partner for amino acid transporters. Pharmacol Ther. 2010;128:119-28.

7. Gheblawi M, Wang K, Viveiros A, Nguyen Q, Zhong JC, Turner AJ, et al. Angiotensin-converting enzyme 2: SARS-CoV-2 receptor and regulator of the renin-angiotensin system: celebrating the 20th anniversary of the discovery of ACE2. Circ Res. 2020;126:1456-74.

8. Towler P, Staker B, Prasad SG, Menon S, Tang J, Parsons T, et al. ACE2 X-ray structures reveal a large hinge-bending motion important for inhibitor binding and catalysis. J Biol Chem. 2004;279:17996-8007.

9. Kuba K, Imai Y, Rao S, Gao H, Guo F, Guan B, et al. A crucial role of angiotensin converting enzyme 2 (ACE2) in SARS coronavirus-induced lung injury. Nat Med. 2005;11:875-9.

10. Zhou $P$, Yang $X-L$, Wang $X-G, H u$, Zhang $L$, Zhang $W$, et al. A pneumonia outbreak asso-ciated with a new coronavirus of probable bat origin. Nature. 2020;579:270-3.

11. Ziegler CGK, Allon SJ, Nyquist SK, Mbano IM, Miao VN, Tzouanas CN, et al. SARSCoV-2 receptor ACE2 is an interferon-stimulated gene in human airway epithelial cells and is detected in specific cell subsets across tissues. Cell. 2020;181: P1016-1035.

12. Lu J, Sun PD. High affinity binding of SARS-CoV-2 spike protein enhances ACE2 carboxypeptidase activity. J Biol Chem. 2020;295:18579-88.

13. Heurich A, Hofmann-Winkler H, Gierer S, Liepold T, Jahn O, Pöhlmann S. TMPRSS2 and ADAM17 cleave ACE2 differentially and only proteolysis by TMPRSS2 augments entry driven by the severe acute respiratory syndrome coronavirus spike protein. J Virol. 2014;88:1293-307.

14. Lambert DW, Yarski M, Warner FJ, Thornhill P, Parkin ET, Smith Al, et al. Tumor necrosis factor-alpha convertase (ADAM17) mediates regulated ectodomain shedding of the severe-acute respiratory syndrome-coronavirus (SARS-CoV) receptor, angiotensin-converting enzyme-2 (ACE2). J Biol Chem. 2005;280:30113-9.

15. Wallentin L, Lindbäck J, Eriksson N, Hijazi Z, Eikelboom JW, Ezekowitz MD, et al. Angiotensin converting enzyme 2 (ACE2) levels in relation to risk factors for COVID-19 in two large cohorts of patients with atrial fibrillation. Eur Heart J. 2020;41:4037-46.

16. Kornilov SA, Lucas I, Jade K, Dai CL, Lovejoy JC, Magis AT. Plasma levels of soluble ACE2are associated with sex, Metabolic Syndrome, and its biomarkers in a large cohort, pointing to a possible mechanism for increased severity in COVID-19. Crit Care. 2020;24:452.

17. Wu J, Deng W, Li S, Yang X. Advances in research on ACE2 as a receptor for 2019nCoV. Cell Mol Life Sci. 2020;11:1-14.

18. Imai Y, Kuba K, Rao S, Huan Y, Guo F, Guan B, et al. Angiotensin-converting enzyme 2 protects from severe acute lung failure. Nature. 2005;436:112-6.

19. Khan A, Benthin C, Zeno B, Albertson TE, Boyd J, Christie JD, et al. A pilot clinical trial of recombinant human angiotensin-converting enzyme 2 in acute respiratory distress syndrome. Crit Care. 2017;21:234.

20. Zoufaly A, Poglitsch M, Aberle JH, Hoepler W, Seitz T, Traugott M, et al. Human recombinant soluble ACE2 in severe COVID-19. Lancet Respir Med. 2020;8: P1154-1158.

21. Epelman S, Tang WH, Chen SY, Lente FV, Francis GS, Sen S. Detection of soluble angiotensin-converting enzyme 2 in heart failure: insights into the endoge-nous counter-regulatory pathway of the renin-angiotensin-aldosterone system. J Am Coll Cardiol. 2008;52:750-4.

22. Iwanaga N, Cooper L, Rong L, Beddingfield B, Crabtree J, Tripp RA, et al. Novel ACE2-IgG1 fusions with improved activity against SARS-CoV2. bioRxiv. 2020. https://doi.org/10.1101/2020.06.15.152157.

23. Xiao T, Lu J, Zhang J, Johnson RI, McKay LGA, Storm N, et al. A trimeric human angiotensin-converting enzyme 2 as an anti-SARS-CoV-2 agent. Nat Struct Mol Biol. 2021;28:202-9.

24. Wan Y, Shang J, Sun S, Tai W, Chen J, Geng Q, et al. Molecular mechanism for antibody-dependent enhancement of coronavirus entry. J Virol. 2020;94: e02015-19.

25. Wysocki J, Ye M, Hassler L, Gupta AK, Wang Y, Ni-coleascu V, et al. A novel soluble ACE2 variant with prolonged duration of action neutralizes SARS-CoV-2 infection in human kidney organoids. J Am Soc Nephrol. 2021;32:795-803.

26. Hassler L, Wysocki J, Gelarden I, Tomatsidou A, Gula H, Nicoleascu V, et al. A novel soluble ACE2 protein totally protects from lethal disease caused by SARS-CoV-2 infection. bioRxiv. 2021. https://doi.org/10.1101/2021.03.12.435191.

27. Wu D, Lewis ED, Pae M, Meydani SN. Nutritional modulation of immune function: analysis of evidence, mechanisms, and clinical relevance. Front Immunol. 2019:10:1-19.

28. Fanos V, Pintus MC, Pintus R, Marcialis MA. Lung microbiota in the acute respiratory disease: from coronavirus to metabolomics. J Pediatr Neonat Individ Med. 2020;9:e090139.

29. Hufnagl K, Pali-Schöll I, Roth-Walter F, Jensen-Jarolim E. Dysbiosis of the gut and lung microbiome has a role in asthma. Semin Immunopathol. 2020;42:75-93.

30. Xiao F, Tang M, Zheng X, Liu Y, Li X, Shan H. Evidence for gastrointestinal infection of SARS-CoV-2. Gastroenterology. 2020;158:1831-3.

31. Xu R, Lu R, Zhang T, Wu Q, Cai W, Han X, et al. Temporal association between human upper respiratory and gut bacterial microbiomes during the course of COVID-19 in adults. Commun Biol. 2021;4:240.

32. Zhou X, Chen H, Sheng X, Shen X, Sun Y, Yan J, et al. Engineering probiotics as living diagnostics and therapeutics for improving human health. Microb Cell Factories. 2020;19:56.

33. Barra M, Danino T, Garrido D. Engineered probiotics for detection and treatment of inflammatory intestinal diseases. Front Bioeng Biotechnol. 2020;8:265.

34. Shigemori S, Shimosato T. Applications of genetically modified immunobiotics with high immunoregulatory capacity for treatment of inflammatory bowel diseases. Front Immunol. 2017:8:22.

35. Yang T, Chakraborty S, Saha P, Mell B, et al. Gnoto-biotic rats reveal that gut microbiota regulates colonic mRNA of Ace2, the receptor for SARS-CoV-2 infectivity. Hypertension. 2020;76:e1-e3. 
492

36. Verma A, Xu K, Du T, Zhu P, Liang Z, Liao S, et al. Expression of human ACE2 in Lactobacillus and beneficial effects in diabetic retinopathy in mice. Mol Ther Methods Clin Dev. 2019;14:161-70.

37. Buford TW, Sun Y, Roberts LM, Banerjee A, Peramsetty S, Knighton A, et al. Angiotensin (1-7) delivered orally via probiotic, but not subcutaneously, benefits the gut-brain axis in older rats. GeroScience. 2020;42:1307-21.

\section{ACKNOWLEDGEMENTS}

The author would like to thank National Institute of Technology Sikkim, India.

\section{AUTHOR CONTRIBUTIONS}

BKM is a sole author, who is responsible for set up the structure, writing and editing of whole manuscript.

\section{COMPETING INTERESTS}

The authors declare no competing interests.

\section{ADDITIONAL INFORMATION}

Correspondence and requests for materials should be addressed to Biplab K. Maiti.

Reprints and permission information is available at http://www.nature.com/ reprints

Publisher's note Springer Nature remains neutral with regard to jurisdictional claims in published maps and institutional affiliations. 